\title{
Neutrino Mass and the Higgs Portal Dark Matter in the ESSFSM
}

\author{
Najimuddin Khan (D) ${ }^{1,2}$ \\ ${ }^{1}$ Theoretical Physics Division, Physical Research Laboratory, Ahmedabad 380009, India \\ ${ }^{2}$ Centre for High Energy Physics, Indian Institute of Science, Bangalore 560012, India \\ Correspondence should be addressed to Najimuddin Khan; khanphysics.123@gmail.com
}

Received 20 November 2017; Accepted 8 February 2018; Published 13 March 2018

Academic Editor: Farinaldo Queiroz

Copyright (C) 2018 Najimuddin Khan. This is an open access article distributed under the Creative Commons Attribution License, which permits unrestricted use, distribution, and reproduction in any medium, provided the original work is properly cited. The publication of this article was funded by $\mathrm{SCOAP}^{3}$.

\begin{abstract}
We extend the standard model with three right-handed singlet neutrinos and a real singlet scalar. We impose two $Z_{2}$ and $Z_{2}^{\prime}$ symmetries. We explain the tiny neutrino mass-squared differences with two $Z_{2}$ - and $Z_{2}^{\prime}$-even right-handed neutrinos using type I seesaw mechanism. The $Z_{2}$-odd fermion and the $Z_{2}^{\prime}$-odd scalar can both serve as viable dark matter candidates. We identify new regions in the parameter space which are consistent with relic density of the dark matter from recent direct search experiments LUX-2016 and XENON1T-2017 and LHC data.
\end{abstract}

\section{Introduction}

The found Higgs boson at the Large Hadron Collider (LHC) [1-3] completes the search for the particle content of the standard model (SM). The hierarchy problem related to the Higgs boson mass has motivated a plethora of models such as supersymmetry, and extra dimensions in which the finetuning is reconsidered. However, an inevitable consequence of these models is that the new physics should lie close to the $\mathrm{TeV}$ scale. Nonobservations [4] of any new physics from the collider experiments imply that the Higgs hierarchy issue is reverting back to being an unsolved open problem.

In addition, the $\mathrm{SM}$ is unable to explain some physical phenomena in nature such as the existence of massive neutrinos, the presence of dark matter (DM), the observed matter-antimatter asymmetry, and so forth. In the SM, by construction, the neutrinos are massless as it does not include right-handed neutrinos. However, from the neutrino oscillation experiments, we got convinced that at least two neutrinos have nonzero mass. The neutrino oscillation experiments have given information about the mass-squared differences between neutrino mass eigenstates. However the individual value of the masses is not yet known. It has been seen that the sum of the three neutrino masses is less than $\sim 0.1 \mathrm{eV}$ [57] which is consistent with the cosmological measurements. Individual masses and the basic nature of neutrinos, that is, whether they are Dirac or Majorana particles, are still an open question.

As neutrino masses are very tiny compared to the other fermion masses, it is believed that the mechanism behind neutrino mass generation is different from the other fermions. The other fermions are obtained mass through the Higgs mechanism. The most popular natural explanation of small neutrino masses is the seesaw mechanism. There are broadly three classes of such models, namely, type I, type II, and type III seesaw models requiring involvement of righthanded neutrinos, a $S U(2)_{L}$ triplet scalar with hypercharge $Y=2$ and $S U(2)_{L}$ hyperchargeless triplet fermions, respectively. The minimal scenario in this respect is the canonical type I seesaw mechanism, in which the SM is extended by a right-handed Majorana neutrinos [8-14]. The TeV-scale seesaw mechanism has been discussed in [15-17]. Including extra scalar fields, it has been studied in [18-21].

Various kinds of astrophysical observations, such as anomalies in the galactic rotation curves, gravitational lensing effects in the Bullet cluster, and excess gamma rays (The excess gamma rays from the galactic centers may come from other sources like pulsars.) from the galactic centers, have indicated the existence of DM in the Universe. The cosmological measurements of tiny anisotropies in Cosmic Microwave Background Radiation (CMBR) by the WMAP and Planck Collaboration [5] suggest that the Universe is 
made of $69 \%$ dark energy, $27 \%$ dark matter, and $4 \%$ ordinary matter.

Astrophysical and cosmological data can tell us about the total amount/density of the DM of the Universe. There is still no consensus on what it is composed of and the properties are still unknown. The possibilities of different kinds of baryonic or nonbaryonic DM candidates have been discussed in [22]. The weakly interacting massive particles (WIMPs) are the best viable DM candidates. No evidence of the WIMP has been found from the direct detection experiments such as XENON100 [23], LUX [24, 25], and XENON1T [26]. As these DM-nucleon scattering experiments still have not found any signature in the detector, these experiments have ruled out low mass $(10-50 \mathrm{GeV})$ regions in the parameter space of a $Z$ and Higgs $h$-portal DM. Recent LUX-2016 [25] data has also excluded the mass range $65-550 \mathrm{GeV}$ of a $h$-portal fermionic (it depends on the mixing angle between Higgs and singlet scalar) DM model [27] and scalar DM models [27-29]. It indicates that we may need the multicomponent DM particles to explain the experimental data. We may detect these DMs in the more efficient detector in the future experiments. Multicomponent DM model is needed [30] to explain the Galactic Center gamma ray excess [31] and the colliding galaxy cluster [32-34] simultaneously. Multicomponent DM models have been considered in $[35,36]$ in various models which also include neutrino, Axion, and supersymmetric particles. Various models with two WIMP candidates could lead to typical signatures at different mass scale and have been studied in [37-58].

We add three right-handed $S U(2)$ singlet fermions and a singlet scalar to the SM. We also impose two $Z_{2}$ and $Z_{2}^{\prime}$ symmetries. All SM and the first two fermion fields are even under these $Z_{2}$ and $Z_{2}^{\prime}$ transformations. The Dirac mass terms can be formed using these fermions and the SM neutrinos. We use type I seesaw mechanism to explain the tiny neutrino mass-squared differences and the mixing angles which are observed by the neutrino oscillation experiments. The third $Z_{2}$-odd fermion and $Z_{2}^{\prime}$-odd scalar both can serve as viable DM particles in this work. Moreover, the requisite rate of annihilation is ensured by postulating some $Z_{2}$ and $Z_{2}^{\prime}$ preserving dimension-four and dimension-five operators for the scalar and fermion particles, respectively. The fourpoint interaction term of the extra fermions and scalar can be obtained from other five-dimensional operators [59]. The interaction term of the third fermion and the scalar allows a larger region of the parameter space than what we would have had with a single DM particle (either fermion or scalar) alone. This interplay brings an enriched DM phenomenology compared to the other models having fermion or scalar DM particle. The region of DM masses $65-550 \mathrm{GeV}$ of a fermionic or scalar Higgs portal DM model is excluded from the present LUX experimental data. In this model, we show that the region with masses $50-550 \mathrm{GeV}$ up to $300 \mathrm{TeV}$ is still allowed by the direct search experiments. Hence, we feel a desirable feature of our model for future study.

The plan of the paper is as follows. In Section 2, we present the theoretical framework of our extended singlet scalar fermionic standard model (ESSFSM). We also discuss the
TABLE 1: The $Z_{2} \times Z_{2}^{\prime}$ quantum numbers. $u$ represents the up-type quarks of the three generations $u, c, t$ and $d$ stand for the down-type quarks $d, s, b$. The charged leptons are denoted by $l=e, \mu, \tau$ with the corresponding left-handed neutrinos $\nu_{l}$. $\Phi$ is the SM Higgs doublet. $G^{+}\left(G^{0}\right)$ stand for the charged (neutral) Goldstone boson. $L, R$ stand for left- and right-handed chirality of fermions.

\begin{tabular}{lccc}
\hline Fields & \multicolumn{3}{c}{$\begin{array}{c}\text { Charged under } S U(2) \times Z_{2} \times Z_{2}^{\prime} \\
\text { transformation }\end{array}$} \\
\hline$Q_{L}=\left(\begin{array}{c}u_{L} \\
d_{L}\end{array}\right)$ & $2 U(2)$ & $Z_{2}$ & $Z_{2}^{\prime}$ \\
$u_{R}, d_{R}$ & 1 & 1 & 1 \\
$L=\left(\begin{array}{c}v_{l} \\
l^{-}\end{array}\right)$ & 2 & 1 & 1 \\
$l_{R}$ & 1 & 1 & 1 \\
$\Phi=\left(\begin{array}{c}G^{+} \\
\frac{h+i G^{0}}{\sqrt{2}}\end{array}\right)$ & 2 & 1 & 1 \\
$v_{s, 1}, v_{s, 2}$ & & 1 & 1 \\
$v_{s, 3}$ & 1 & 1 & 1 \\
$S$ & 1 & -1 & 1 \\
\hline
\end{tabular}

diagonalization procedure to get the neutrino mass matrix and the relic density calculation of two dark matter particles. We show the detailed constraints on this model in Section 3. We present our numerical results and show the allowed region in the parameter spaces from the neutrino mass and mixing angle, relic density, and direct detection in Section 4. Finally, we conclude in Section 5.

\section{Theoretical Framework of the Model}

In this section, we give a description of our model. We add three right-handed neutrinos and a scalar to the SM Lagrangian. These extra particles are singlet under $S U(2)$ transformation. We impose two $Z_{2}$ and $Z_{2}^{\prime}$ symmetries such that the SM fields and first two right-handed neutrinos are even under these $Z_{2}$ and $Z_{2}^{\prime}$ transformations. The third righthanded neutrino is odd (even) under $Z_{2}\left(Z_{2}^{\prime}\right)$ transformation whereas the scalar field is even (odd) under $Z_{2}\left(Z_{2}^{\prime}\right)$ transformation. The $Z_{2} \times Z_{2}^{\prime}$ quantum numbers of the SM fields and extra right-handed neutrinos and scalar fields are summarized in Table 1 . The $Z_{2} \times Z_{2}^{\prime}$-even neutrinos are free to mix with the usual SM neutrinos and therefore generate the neutrino masses through type I seesaw mechanism. These symmetries prohibited the coupling of an odd number of the third fermion and/or the scalar particle to the SM particles. The part of Lagrangian that is invariant under $S U(2) \times U(1) \times$ $Z_{2} \times Z_{2}^{\prime}$ transformation is given by

$$
\begin{aligned}
\mathscr{L}= & \frac{i}{2} \bar{\nu}_{s, a} \not \partial \nu_{s, a}-\frac{1}{2} \bar{M}_{\nu_{s, a}} \bar{\nu}_{s, a} \nu_{s, a}^{c}+\frac{1}{2} \partial_{\mu} S \partial^{\mu} S-\frac{\mu_{S}}{2} S^{2} \\
& -\frac{\lambda_{S}}{4 !} S^{4},
\end{aligned}
$$


where summation over $a$ is implied, with $a=1,2,3$ denoting generation indices for the right-handed fermions. $c$ stands for the charge conjugation. The mutual interaction terms of the SM Higgs, left-handed leptons, the extra scalar, and fermions are given by

$$
\begin{aligned}
\mathscr{L}_{\text {mix }}= & -Y_{v, a b} \bar{L}_{a} \Phi^{c} v_{s, b}-\bar{M}_{\nu_{s}, m n} \bar{\nu}_{s, m} \nu_{s, n}^{c}-\frac{\kappa}{2}|\Phi|^{2} S^{2} \\
& +\frac{C_{h, m n}}{\Lambda_{h, m n}}|\Phi|^{2} \bar{\nu}_{s, m} \nu_{s, n}^{c}+\frac{C_{h, a}}{\Lambda_{h, a}}|\Phi|^{2} \bar{\nu}_{s, a} \nu_{s, a}^{c} \\
& +\frac{C_{S, m n}}{\Lambda_{S, m n}} S^{2} \bar{\nu}_{s, m} \nu_{s, n}^{c}+\frac{C_{S, a}}{\Lambda_{S, a}} S^{2} \bar{\nu}_{s, a} \nu_{s, a}^{c}+\text { h.c. }
\end{aligned}
$$

$\Phi$ is the SM Higgs doublet, $\Phi \equiv\left(G^{+},\left(v+h+i G^{0}\right) / \sqrt{2}\right)^{T}$, where $G^{ \pm}$and $G^{0}$ are the Goldstone bosons, and $h$ is the SM Higgs. $\Phi^{c}$ stands for charge conjugate of $\Phi . L \equiv\left(\nu_{l}, l\right)^{T}$ with $l=e, \mu$ and $\tau$ being the left-handed lepton doublet. $b=1,2$ does not assume the third index as the third fermion is odd under $Z_{2}$-symmetry. The indices $m \neq n=1,2$; hence the second term in (2) generates the mixing mass term between two $Z_{2}$ - and $Z_{2}^{\prime}$-even neutrinos. After electroweak (EW) symmetry breaking, the fourth term, that is, the dimensionfive operator also gives an additional mixing mass term. The Higgs to extra neutrinos couplings are also generated from the dimension-five operators (fourth and fifth term in (2)). This will lead to the Higgs boson decay into these extra neutrons. As the $Z_{2}$ - and $Z_{2}^{\prime}$-even neutrinos are considered to be very heavy, the partial decay width of the Higgs to these neutrinos is zero. As we are allowing these dimension-five operators in the Lagrangian, for completeness, we also add the other dimension-five operators $\left(C_{S, m n} / \Lambda_{S, m n}\right) S^{2} \bar{v}_{s, m} \nu_{s, n}^{c}$ and $\left(C_{S, a} / \Lambda_{S, a}\right) S^{2} \bar{\nu}_{s, a} v_{s, a}^{c}$, which in turn give more room in the parameter space to maneuver. In this work, we focus on the dominant dimension-five operators related to the neutrino and Higgs portal dark matter physics, that is, those involving at least one Higgs and neglecting other possible operators which are allowed by the SM gauge and $Z_{2} \times Z_{2}^{\prime}$ symmetries. $\Lambda$ 's are the cut-off scales for the new physics. In our calculation, we assume $\Lambda_{h, a}=\Lambda_{S, a}=\Lambda_{h, m n}=$ $\Lambda_{S, m n} \equiv \Lambda . C_{h, a}, C_{S, a}, C_{h, m n}$, and $C_{S, m n}$ are dimensionless coupling parameters. The cut-off scale $\Lambda$ and $C_{h, 12}$ and the Yukawa couplings $Y_{v, a b}$ are important to explain the neutrino oscillation observables, whereas $\Lambda, C_{h, 3}, C_{S, 3}$, and $\kappa$ could change the masses and coupling strength of DM particles to the Higgs. In addition, these could alter the self-annihilation interaction probability of the heavier DM particles into the lighter DM particles. Hence, these parameters play a crucial role to calculate the relic density of the DM particles $v_{s, 3}$ and $S$. The masses of the DM particles are given by

$$
\begin{aligned}
M_{v_{s, 3}} & =\bar{M}_{v_{s, 3}}-\frac{C_{h, 3}}{\Lambda} v^{2}, \\
M_{S}^{2} & =\mu_{S}^{2}+\frac{1}{2} \kappa v^{2},
\end{aligned}
$$

and the coupling strength of the DM candidates with the Higgs can be written as

$$
h \bar{\nu}_{s, 3} \nu_{s, 3}: \frac{C_{h, 3}}{\Lambda}
$$

$$
h S S: \frac{\kappa}{2} v
$$

The parameter $C_{S, 3}$ is responsible for the annihilation of $S S \leftrightarrow \bar{\nu}_{s, 3} \nu_{s, 3}$. This process reduces the number density of the heavier DM till the freeze-out.

It is also important to note that the gauge boson $B_{\mu}$ and/or $W_{\mu}^{i}$ interactions terms are not present in the kinetic part of the Lagrangian (see (1)). Therefore, this model does not have any extra gauge-boson contribution to the DM-nucleus scattering cross section which is allowing larger region in the parameter space from the direct detection experiments. This is the specialty of the presence of real singlet scalar and fermion in the ESSFSM.

2.1. Diagonalization Procedure of Type I Seesaw Matrix and Nonunitarity of PMNS Matrix. Here, we show the diagonalization procedure $[60,61]$ of type I seesaw mechanism to generate tiny neutrino mass-squared difference $[62,63]$. In this model, $5 \times 5$ neutrino mass matrix in the basis $\left(\nu_{l}, v_{s}\right)$ can be written as

$$
M_{v}=\left(\begin{array}{cc}
0 & M_{D} \\
M_{D}^{T} & M_{v_{s}}
\end{array}\right),
$$

where the Dirac mass $M_{D}$ and Majorana mass $M_{v_{s}}$ terms can be written as

$$
\begin{aligned}
M_{D} & =\left(\begin{array}{ll}
Y_{v, 11} v & Y_{v, 12} v \\
Y_{v, 21} v & Y_{v, 22} v \\
Y_{v, 31} v & Y_{v, 32} v
\end{array}\right), \\
M_{v_{s}} & =\left(\begin{array}{ll}
M_{11} & M_{12} \\
M_{12} & M_{22}
\end{array}\right) .
\end{aligned}
$$

Here, $M_{11}=\bar{M}_{v_{s}, 1}-\left(C_{h, 1} / \Lambda\right) v^{2}, M_{22}=\bar{M}_{v_{s}, 2}-\left(C_{h, 2} / \Lambda\right) v^{2}$, and $M_{12}=\bar{M}_{v_{s}, 12}-\left(C_{h, 12} / \Lambda\right) v^{2}$

Using $5 \times 5$ unitary matrices $[64,65]$, one can diagonalize the neutrino mass matrix $M_{v}$ in (5). It is given by

$$
U^{T} M_{\nu} U=M_{\nu}^{\text {diag }},
$$

where $M_{v}^{\text {diag }}=\operatorname{diag}\left(m_{i}, M_{j}\right)$ with mass eigenvalues $m_{i}(i=$ $1,2,3)$ for three light neutrinos and $M_{j}(j=1,2)$ for two heavy neutrinos, respectively. In this calculation, we have two nonzero mass eigenstates of light neutrinos. We consider $m_{1}$ to be zero. In the limit $M_{D}^{2} \ll M_{v_{s}}^{2}$, the matrix $U$ can be expressed as follows [61]:

$$
\begin{aligned}
U & =W T=\left(\begin{array}{cc}
U_{L} & V \\
S & U_{H}
\end{array}\right) \\
& =\left(\begin{array}{cc}
\left(1-\frac{1}{2} \epsilon\right) U_{v} & M_{D}^{*}\left(M_{v_{s}}^{-1}\right)^{*} U_{R} \\
-M_{v_{s}}^{-1} M_{D}^{T} U_{v} & \left(1-\frac{1}{2} \epsilon^{\prime}\right) U_{R}
\end{array}\right),
\end{aligned}
$$


where $U_{L}, V, S$ and $U_{H}$ are $3 \times 3,2 \times 3,3 \times 2$ and $2 \times 2$ matrices, respectively, which are not unitary. The unitary $W$ matrix which brings the full $5 \times 5$ neutrino matrix in the block diagonal forms as

$$
W^{T}\left(\begin{array}{cc}
0 & \widehat{M}_{D} \\
M_{D}^{T} & M_{v_{s}}
\end{array}\right) W=\left(\begin{array}{cc}
m_{\text {light }} & 0 \\
0 & M_{\text {heavy }}
\end{array}\right) .
$$

Another unitary matrix $T=\operatorname{diag}\left(U_{v}, U_{R}\right)$ matrix again diagonalizes the mass matrices in the light and heavy sectors are appearing in the upper and lower block of the block diagonal matrix, respectively. In the above-stated limit, one can then write the light neutrino mass matrix to the leading order as

$$
m_{\text {light }}=M_{D} M_{v_{s}}^{-1} M_{D}^{T} \text {. }
$$

In (8), $U_{L}$ corresponds to the PMNS matrix which acquires a nonunitary correction $(1-\epsilon / 2)$ due to the presence of heavy neutrinos. The characterizations of nonunitarity are denoted by the notations $\epsilon$ and $\epsilon^{\prime}$. These are given by the following [60]:

$$
\begin{aligned}
\epsilon & =M_{D}^{*} M_{\nu_{s}}^{-1 *} M_{\nu_{s}}^{-1} M_{D^{\prime}}^{T} \\
\epsilon^{\prime} & =M_{\nu_{s}}^{-1} M_{D}^{T} M_{D}^{*} M_{\gamma_{s}}^{-1 *} .
\end{aligned}
$$

2.2. Relic Density Calculation of the Two-Component Dark Matter. In order to calculate the relic abundance of twocomponent DM in the present formalism, we need to solve the relevant coupled Boltzmann equations [66]:

$$
\begin{aligned}
\frac{d n_{v_{s, 3}}}{d t}+ & 3 H n_{v_{s, 3}} \\
= & -\langle\sigma v\rangle_{v_{s, 3} v_{s, 3} \rightarrow X X}\left(n_{v_{s, 3}^{2}}^{2} n_{v_{s, 3} \mathrm{eq}}^{2}\right) \\
& -\langle\sigma v\rangle_{v_{s, 3} v_{s, 3} \rightarrow S S}\left(n_{v_{s, 3}}^{2}-\frac{n_{v_{s, 3} \mathrm{eq}}^{2}}{n_{S_{\mathrm{eq}}}^{2}} n_{S}^{2}\right), \\
\frac{d n_{S}}{d t}+ & 3 H n_{S} \\
= & -\langle\sigma v\rangle_{S S \rightarrow X X}\left(n_{S}^{2}-n_{S_{\mathrm{eq}}}^{2}\right) \\
& -\langle\sigma v\rangle_{S S \rightarrow v_{s, 3} v_{s, 3}}\left(n_{S}^{2}-\frac{n_{S_{\mathrm{eq}}}^{2}}{n_{v_{s, 3} \mathrm{eq}}^{2}} n_{v_{s, 3}^{2}}^{2}\right),
\end{aligned}
$$

where $Z_{2}$-even (SM, $v_{s, 2}$ and $v_{s, 2}$ ) particles are denoted by $X$. In addition, the heavier $X$ can decay into lighter particles. $\langle\sigma v\rangle$ is the average effective annihilation cross sections of the DM candidates which include all $n \geq 2$-body final state particles. The first term on the right-hand side of (12) indicates the contribution of annihilation to SM particles whereas the second term in both equations takes care of the contribution of the self-scattering of DM particles. The contributions from the processes $v_{s, 3} S \rightarrow v_{s, 3} S$ are zero as it does not alter the number density. In the very early Universe, both of the DM candidates are in thermal and chemical equilibrium. In the nonrelativistic case, if the temperature $T$ of the Universe is less than the DM masses, then the equilibrium number density takes the form $n_{\mathrm{DM} \text { eq }}=\left(M_{\mathrm{DM}} T / 2 \pi\right)^{3 / 2} \exp \left(-M_{\mathrm{DM}} / T\right)$. As the temperature was falling down, some species are decoupled and contributing to the relic density. The heavier DM candidate particle decouples earlier than the lighter one. In the present Universe, they both were frozen out and giving a partial contribution in the total relic abundance $\Omega_{\text {tot }}$. If the individual contributions of the fermion and scalar are $\Omega_{v_{s, 3}}$ and $\Omega_{S}$, then the total relic abundance $\Omega_{\text {tot }}$ can be written as

$$
\Omega_{\mathrm{DM}}=\Omega_{v_{s, 3}}+\Omega_{S}
$$

where $\Omega_{v_{s, 3}}=\left(M_{v_{s, 3}} / \rho_{c}\right) n_{v_{s, 3}}\left(T_{0}\right)$ and $\Omega_{v_{s, 3}}=\left(M_{S} / \rho_{c}\right) n_{S}\left(T_{0}\right)$. $\rho_{c} \sim 1.05 \times 10^{-5} h^{2} \mathrm{GeVcm}^{-3}$ stands for the critical density of the present Universe; $h=0.72$ is the Hubble parameter. $n\left(T_{0}\right)$ is the number density of the DM at temperature $T_{0}$ today.

One can note that if the masses of the DM particles are degenerate, then the Boltzmann equations (12) become decoupled; that is, self-scattering cross sections of the process $v_{s, 3} v_{s, 3} \leftrightarrow S S$ are very small compared to the self-annihilation cross section of the DM. These equations describe the evolution of each DM independently. In our calculation, we use the mi crOMEGAs [66] and solve the above coupled Boltzmann equations to calculate the individual number density of the DM particles in the present Universe.

\section{Constraints on the Model}

The parameter spaces of this model are constrained from various theoretical considerations like absolute vacuum stability, perturbation, and unitarity of the scattering matrix. The absolute stability of the Higgs potential demands that the scalar potential should not approach to negative infinity along any direction of the field space at large field values. The required conditions are $\lambda>0, \lambda_{S}>0$, and $\kappa>-\sqrt{2 \lambda \lambda_{s}} / \sqrt{2}$, where $\lambda$ is the Higgs quartic coupling [29]. Lagrangian of our model remains perturbative $[67,68]$ for $|\lambda| \lesssim 4 \pi / 3,|\kappa| \lesssim$ $8 \pi,\left|\lambda_{S}(\Lambda)\right| \lesssim 8 \pi, C_{h, a} \lesssim 8 \pi$, and $C_{S, a} \lesssim 8 \pi$. The parameters of the scalar part of Lagrangian (see (1) and (2)) of this model are constrained by the unitarity of the scattering matrix (Smatrix). One can obtain the $\mathrm{S}$-matrix by using various scalarscalar, gauge boson-gauge boson, and scalar-gauge boson scattering amplitudes. We use the equivalence theorem [6971] to reproduce the S-matrix for this model [68]. The unitary bounds demand that the eigenvalues of this matrix should be less than $8 \pi$ which imply $\lambda \leq 8 \pi$ and $\mid 12 \lambda+\lambda_{S} \pm$ $\sqrt{16 \kappa^{2}+\left(-12 \lambda+\lambda_{S}\right)^{2}} \mid \leq 32 \pi$.

The observed neutrino mass-squared differences and mixing angles by the neutrino oscillation experiments put stringent constraints on the parameter space of this model. The Higgs signal strength and the decay width measured by the LHC, the relic density, and direct-indirect searches of DM all alone restrict the allowed parameter space considerably. We discuss these in the following.

3.1. Bounds from the Neutral Fermion Mass and Mixing Angles. The global analysis of neutrino oscillation measurements 
provides the neutrino oscillation parameters for both normal and inverted hierarchies scenario. These can be found in $[62,63]$. The measurements of the electroweak precision observables along with other experimental data put severe constraints on the light neutrino mixing matrix $U_{L}$. The detailed analysis has been given in Refs [72, 73].

The L3 collaboration at the LEP had analyzed the decay channels $N \rightarrow e^{ \pm} W^{\mp}$ to find the evidence of the heavy neutrino. No signature had been found for the mass range in between $80 \mathrm{GeV}$ ( with $\left|V_{\alpha i}\right|^{2} \leq 2 \times 10^{-5}$ ) and $205 \mathrm{GeV}$ ( with $\left.\left|V_{\alpha i}\right|^{2} \leq 1\right)$ [74]. $V$ is the light-heavy mixing matrix, given in (8). This puts a lower bound on the mass of the heavy neutrino and the mixing matrix elements $V_{\alpha i} \cdot\left|V_{\alpha i}\right|^{2} \gtrsim 10^{-5}$ and $3<M_{1,2}<M_{Z}$ regions have also been ruled out from the invisible decay width of the $Z$-boson [75-77].

The experimental data [78] $\operatorname{Br}(\mu \rightarrow e \gamma)<4.2 \times 10^{-13}$ of the flavor changing decay processes has restricted the arbitrary Yukawa coupling $Y_{v}$. In this model, the branching ratio can be written as follows [79]:

$$
\operatorname{Br}(\mu \longrightarrow e \gamma)=\frac{3 \alpha}{8 \pi}\left|V_{e i} V_{i \mu}^{\dagger} f(x)\right|^{2},
$$

where $x=\left(M_{i}^{2} / M_{W}^{2}\right), i=1,2$, stands for the mass of heavy neutrinos and $f(x)$ is the slowly varying function can be found in [79].

3.2. Bounds from the Higgs Signal Strength at the LHC. The dominant contribution of the Higgs $h$-production cross section is coming through the gluon fusion. In this work, the Higgs to diphoton signal strength $\mu_{\gamma \gamma}$ can be written as

$$
\begin{aligned}
\mu_{\gamma \gamma} \simeq & \frac{\sigma(g g \rightarrow h \rightarrow \gamma \gamma)_{\mathrm{ESSFSM}}}{\sigma(g g \rightarrow h \rightarrow \gamma \gamma)_{\mathrm{SM}}} \\
= & \frac{\sigma(g g \rightarrow h)_{\mathrm{ESSFSM}}}{\sigma(g g \rightarrow h)_{\mathrm{SM}}} \frac{\operatorname{Br}(h \rightarrow \gamma \gamma)_{\mathrm{ESSFSM}}}{\mathrm{Br}(h \rightarrow \gamma \gamma)_{\mathrm{SM}}}
\end{aligned}
$$

The production cross section of $h$ is the same as in the SM. Then $\mu_{\gamma \gamma}$ can be written as

$$
\mu_{\gamma \gamma}=\frac{\Gamma_{h, \mathrm{SM}}^{\mathrm{total}}}{\Gamma_{h, \mathrm{ESSFM}}^{\mathrm{total}}}, \quad \text { as } \frac{\Gamma_{h}^{\mathrm{total}}}{M_{h}} \longrightarrow 0 .
$$

As we do not have any extra charged particle, the decay width $\Gamma(h \rightarrow \gamma \gamma)$ is the same as in the SM. If the extra particles (scalar and fermions) have the mass less than half of the Higgs mass $M_{h} / 2$, then the diphoton signal strength could be changed due to the invisible decay of the Higgs boson. Using the global fit analysis [80] that such an invisible branching ratio is less than $\sim 20 \%$, so the decay width in (16) provides a suppression of about $\sim 80-100$ percent. The present combined value of $\mu_{\gamma \gamma}$ by the ATLAS and CMS collaborations is $1.14_{-0.18}^{+0.19}$ [81]. As the partial decay width of the Higgs to the heavy $Z_{2}$ - and $Z_{2}^{\prime}$-even neutrinos is zero, it cannot alter $\mu_{\gamma \gamma}$. We also check that the mass region $M_{\mathrm{DM}}<55 \mathrm{GeV}$ of the $Z_{2^{-}}^{\prime}$ odd scalar and $Z_{2}$-odd neutrino DM along with $|\kappa| \geq 0.004$ and/or $\left|C_{h, 3}\right| \geq 0.2$ is excluded at $2 \sigma$.
3.3. Relic Density and Direct Search Limits. The relic density of DM all alone restricts the allowed parameter space. The parameter space of this model should also satisfy the combined WMAP and Planck [82] imposed dark matter relic density constraint $\Omega_{\text {tot }} h^{2}=0.1198 \pm 0.0026$. In our calculation, we use the micrOMEGAs [66] to calculate the total relic density of the two DM particles. In this model, we find the correct relic density for the dark matter particles mass $M_{\mathrm{DM}}<55 \mathrm{GeV}$. However, these regions in the parameter spaces are ruled out from the invisible Higgs decay width and direct search data. In the following, we discuss the detailed constraints from direct detection of two-component dark matter particles.

The WIMPs, in particular, those that have nonvanishing weak interactions with the SM and therefore can be tested. They are actively being searched for in the direct detection experiments which look for their nuclear scatterings in the deep underground detectors. If the DM scatters from atomic nucleus, then it leaves their signature in form of a recoiled nucleus. However, no confirmed detection of the DM in the experimental laboratory has been made so far. If a discovery is within the reach of a near-future direct detection experiment, then these experiments will be able to constrain the WIMP properties such as its mass, DM-nucleus scattering cross section, and possibly spin.

As we have two-component DM, it is very difficult to distinguish these DM particles in the direct detection experiment. The local number density of the DM particles in the solar neighborhood is important in determining the total number of event rate in the experiment. It is not entirely straightforward to determine which component dominates the event rate. There have been only a few works regarding the direct detection of multicomponent DM [83-85]. The signal rate generated from two-component DM in the detector is different compared to a single-component DM and it completely depends on the DM masses and local densities in the solar neighborhood. The particle masses will determine their individual rates (see section 3.2 of [85]) that can distinguish one- or two-component DM if the DM particles have different masses.

Presently, nonobservation of DM in the direct detection experiments such as XENON100 [23], LUX [24, 25], and XENON1T [26] sets a limit on WIMP-nucleon scattering cross section for given DM masses. The most stringent bound is set by the XENON1T [26] and LUX 2016 [25] exclusion data. The region above the green line in Figure 2 is excluded. We translate the LUX exclusion data into some allowed or excluded zones in the parameter spaces of our model comprising $C_{h, 3}, M_{v_{s, 3}}, \kappa, M_{S}$ and $C_{S, 3}$. In this model, the Feynman diagrams for the scattering of DM particles $v_{s, 3}, S$ with the nuclei are shown in Figure 1. In the limit $M_{\mathrm{DM}}\left(M_{\gamma_{s, 3}}, M_{S}\right) \gg M_{N}$, the fermion-nucleon and scalarnucleon scattering cross sections are roughly given by

$$
\begin{aligned}
\sigma_{v_{s, 3}, N} & =X_{N}\left(\frac{C_{h, 3}}{\Lambda M_{v_{s, 3}}}\right)^{2}, \\
\sigma_{S, N} & =\frac{X_{N}}{2}\left(\frac{\kappa}{M_{S}}\right)^{2},
\end{aligned}
$$




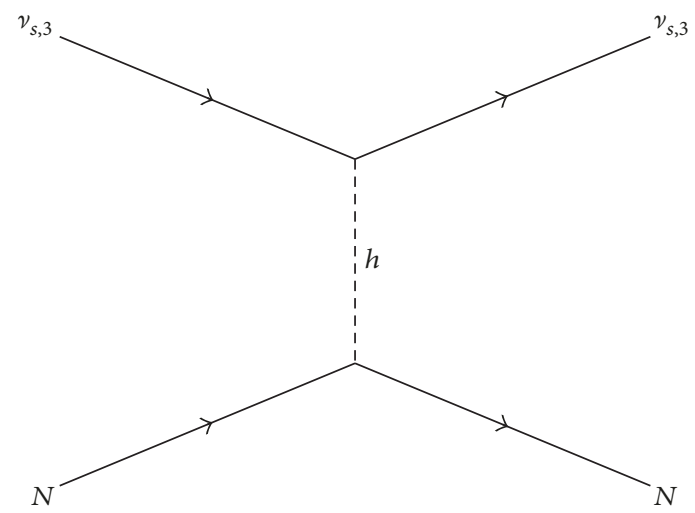

(a)

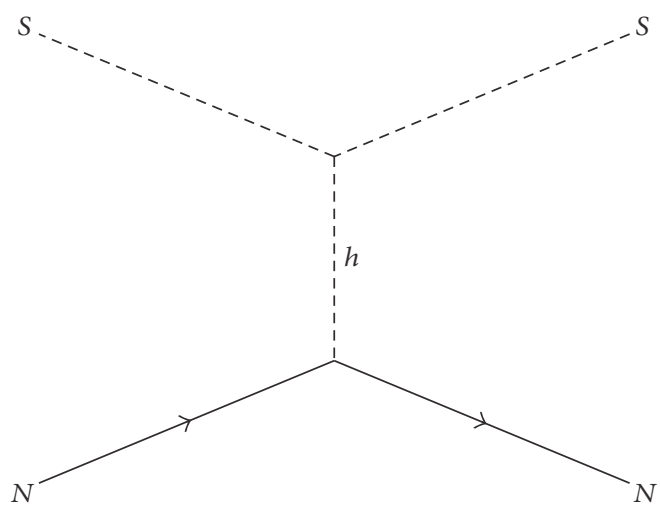

(b)

FIgURE 1: (a) Lowest order Feynman diagram for singlet neutrino-nucleus elastic scattering via the Higgs mediation. (b) A similar diagram for the singlet scalar-nucleus elastic scattering.

where $X_{N}=\left(m_{r} m_{N} f / \sqrt{\pi} M_{h}^{2}\right)^{2}$ and $f \approx 0.3$ is the form factor of the nucleus. $m_{r}$ represents the reduced mass of the nucleus and the scattered DM particle.

Using (17), we calculate the DM-nucleon cross sections for the two dark matter components of different mass. The region in the parameter space for which DM-nucleon cross section falls above the green line in Figure 2 is ruled out by the recent LUX-2016 [25] exclusion data. The region above the purple line is ruled out by the recent XENON-2017 [26] data.

\section{Numerical Results}

We explain the neutrino mass using type I seesaw mechanism. We show that our results are compatible with the various constraints such as the charged lepton flavor violating decay $\mu \rightarrow e \gamma$. In addition, the extra $Z_{2}$-odd fermion and the $Z_{2}^{\prime}$-odd scalar both can serve as viable DM particles producing the relic density in the right ballpark. We show that the regions in the parameter space are consistent with the Planck/WMAP as well as LUX-2016 and XENON-2017 data. In this study, we use FeynRules [86] along with micrOMEGAs [66] to compute the relic density of the DM candidates $v_{s, 3}$ and $S$. We will discuss these in the following.

4.1. Neutrino Oscillation Parameters. We obtain tiny neutrino mass through type I seesaw mechanism. We use the input parameters such as the new Yukawa couplings $Y_{\nu, i j}(i=1,2,3$ and $j=1,2$ ), dimensionless couplings $C_{h, 1}, C_{h, 2}, C_{h, 12}$, and the mass terms $\bar{M}_{v_{s}, 1}, \bar{M}_{v_{s}, 2}$, and $\bar{M}_{v_{s}, 12}$. In our calculation, we assume cut-off scale for the new physics is $\Lambda=10 \mathrm{TeV}$. In order to explain successful leptogenesis [87, 88], we need complex Yukawa coupling to have nonzero CP-violation. The detailed discussion can be found in [61]. The presence of the extra Majorana neutrinos will allow for neutrinoless double $\beta$-decay [12]. In this work, we use the nonzero and real Yukawa couplings $Y_{v, 12}=Y_{v, 23}\left(\equiv y_{v}\right)$. Other Yukawa couplings are taken to zero. We chose the values of parameters $\bar{M}_{\gamma_{s}, 12}$ and $C_{h, 12}$ such that the off-diagonal components of the heavy mass matrix $M_{v_{s}}$ become zero (see (6)). We consider three heavy neutrino masses $\mathcal{O}\left(10^{11}\right) \mathrm{GeV}, \mathcal{O}\left(10^{5}\right) \mathrm{GeV}$, and $\mathcal{O}\left(10^{3}\right) \mathrm{GeV}$ and the corresponding three different Yukawa couplings $y_{v}$ to obtain tiny the neutrino masses. We present these benchmark points and the corresponding low-energy variables in Table 2 . These variables are consistent with the experimental data. As the cut-off scale for the new physics $\Lambda$ is very large, the dimensionless couplings $C_{h}$ (within perturbative limit) could not alter the neutrino mass considerably.

4.2. New Regions in the DM Parameter Space. We have seen that the region in the parameter space $M_{\mathrm{DM}}<\mathcal{O}(500) \mathrm{GeV}$ of a single Higgs portal WIMP DM particle is ruled out by the recent LUX experiment. Hence, it becomes important to show that these regions in the parameter space are still alive in the ESSFSM. In Table 3, we present five benchmark points for this model which are producing right relic density and allowed by the recent nonobservation of DM-nucleon scattering in the LUX experiment. The DM mass regions below the half of the Higgs mass are also consistent with the Higgs invisible decay width [80]. If the mass difference between the fermionic and scalar DM particles is very large, then it is expected that lighter one will dominate over the heavier one in contributing to the relic density. For $M_{v_{s, 3}} \simeq$ $M_{S}$ and tiny interaction coupling $C_{S, 3}$, the contribution of these DM particles is nearly equal to the total relic density, whereas the interaction coupling $C_{S, 3} \sim \mathbf{O}(1)$ and a huge mass difference in the DM particles with particular Higgs portal couplings $\kappa$ and $C_{h, 3}$ can produce equal relic density in the Universe. For example, see benchmark points I-IV. The lighter DM mass near $60 \mathrm{GeV}$ will always dominate over the heavier one because the contribution of self-annihilation processes $\mathrm{DM}, \mathrm{DM} \rightarrow b \bar{b}$ into the relic density is larger than the other processes. The other processes can dominate over the DM, DM $\rightarrow b \bar{b}$ process for the choice of the large Higgs portal coupling $\kappa$. In this case, the relic density and the direct detection data restrict such a choice of $\kappa$. 
TABLE 2: Three lists of benchmark points used in our analysis. Using these BPs, we have obtained the outputs for our model which are satisfying all the low energy constraints.

\begin{tabular}{|c|c|c|c|}
\hline \multirow{2}{*}{ Parameters } & \multicolumn{3}{|c|}{ Benchmark points for $Z_{2}$ - and $Z_{2}^{\prime}$-even fermions } \\
\hline & BM-I & BM-II & BM-III \\
\hline$M_{11} \mathrm{GeV}$ & $1.2 \times 10^{11}$ & $7.1 \times 10^{5}$ & $6.9 \times 10^{3}$ \\
\hline$C_{h, 1}$ & 0.1 & 0.01 & 0 \\
\hline$M_{22} \mathrm{GeV}$ & $1.4 \times 10^{12}$ & $2.36 \times 10^{5}$ & $2.41 \times 10^{3}$ \\
\hline$C_{h, 2}$ & 0.1 & 0.01 & 0 \\
\hline$M_{12} \mathrm{GeV}$ & 0 & 0 & 0 \\
\hline$y_{v}$ & 0.01 & $10^{-5}$ & $10^{-6}$ \\
\hline Outputs & \multicolumn{3}{|c|}{ Corresponding low-energy variables } \\
\hline$\Delta m_{21}^{2} / 10^{-5} \mathrm{eV}^{2}$ & 7.5001 & 7.2909 & 7.7197 \\
\hline$\Delta m_{31}^{2} / 10^{-3} \mathrm{eV}^{2}$ & 2.55234 & 2.63959 & 2.5312 \\
\hline$\theta_{12}$ & 0.5883 & 0.5774 & 0.5720 \\
\hline$\theta_{23}$ & 0.7953 & 0.7854 & 0.7803 \\
\hline$\theta_{13}$ & 0.1476 & 0.1473 & 0.1469 \\
\hline$\delta_{\text {PMNS }} \mathrm{rad}$ & $10^{-5}$ & $10^{-4}$ & $10^{-3}$ \\
\hline$\alpha \mathrm{rad}$ & 1.7 & 1.8 & 1.9 \\
\hline$m_{i} \mathrm{eV}$ & $0,0.0087,0.0505$ & $0,0.0085,0.0514$ & $0,0.0088,0.0505$ \\
\hline $\operatorname{Br}(\mu \rightarrow e \gamma)$ & $3.0 \times 10^{-48}$ & $1.9 \times 10^{-37}$ & $1.69 \times 10^{-33}$ \\
\hline
\end{tabular}

TABLE 3: Lists of benchmark points used in our analysis. Using these BPs we obtain the relic density in the right ballpark allowed by LUX-2016 direct detection data.

\begin{tabular}{|c|c|c|c|c|c|c|c|c|c|c|}
\hline \multirow{2}{*}{ Benchmark points } & \multicolumn{5}{|c|}{ Parameters } & \multirow{2}{*}{ Relic density $\Omega h^{2}$} & \multicolumn{2}{|c|}{ Percentage of DM } & \multicolumn{2}{|c|}{ DM-N cross section in [zb] } \\
\hline & $M_{\gamma_{s, 3}} \mathrm{GeV}$ & $C_{h, 3}$ & $M_{S} \mathrm{GeV}$ & $\kappa$ & $C_{S, 3}$ & & Fermion & Scalar & Fermion & Scalar \\
\hline BP-I & 260 & 0.05 & 59 & 0.0015 & 0.1 & 0.1271 & 39.12 & 61.88 & 0.33 & 0.0054 \\
\hline BP-II & 130 & 0.01 & 60 & 0.001 & 0.1 & 0.1263 & 40.68 & 59.32 & 0.013 & 0.0023 \\
\hline BP-III & 86 & -0.01 & 59.8 & 0.0012 & 0.1 & 0.1129 & 53.10 & 46.90 & 0.013 & 0.0033 \\
\hline BP-IV & 62 & -0.01 & 60.9 & 0.0016 & 0.1 & 0.1156 & 69.76 & 30.24 & 0.013 & 0.0058 \\
\hline BP-V & 59 & 0.02 & 250 & 0.0025 & 0.1 & 0.1201 & 99.6 & 0.4 & 0.053 & 0.0008 \\
\hline
\end{tabular}

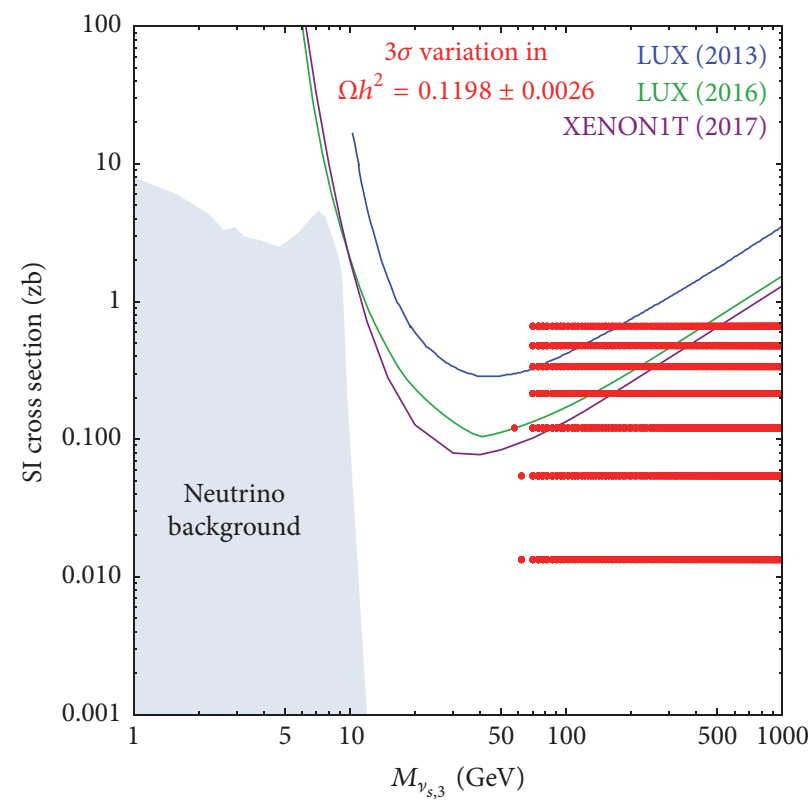

(a)

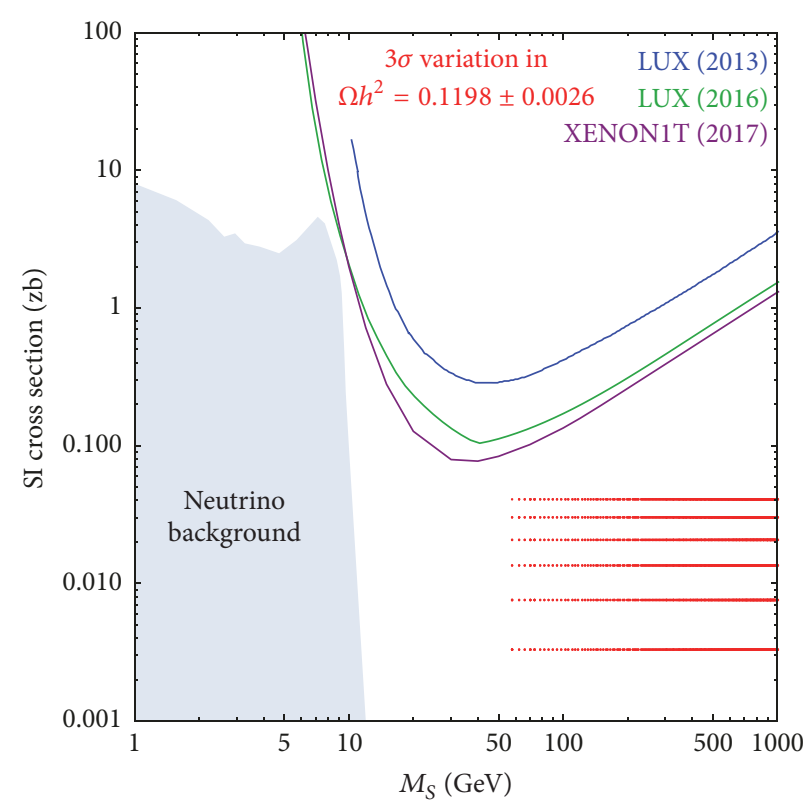

(b)

FIgURE 2: WIMP-nucleon cross section versus DM mass keeping $C_{S, 3}=0.1$. The gray region indicates the neutrino background. Note that each plot contains only $10^{7}$ data (red) points. Larger data points can fill the gap between the red bands. 


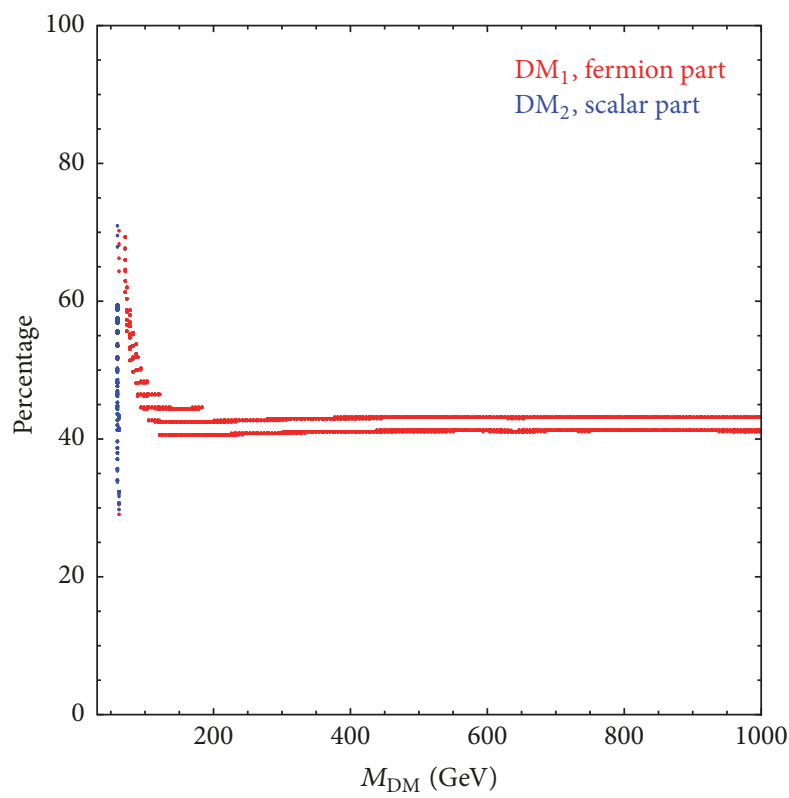

(a)

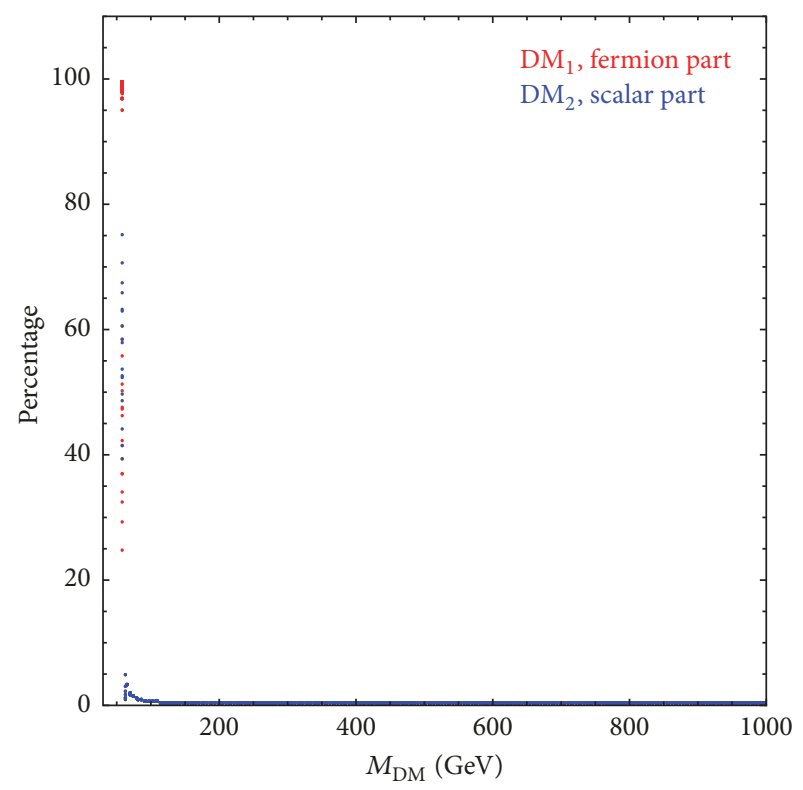

(b)

FIGURE 3: Percentage of DM contributing to the total relic density. Red points correspond to the fermionic DM contributions whereas blue points correspond to the singlet scalar.

In order to find the favored regions in the parameter space which satisfies DM relic density constraints and the recent LUX direct detection data, in Figure 2, we present two contour plots of relic density $\Omega h^{2}$ in the DM-nucleon cross section versus mass plane. The red points are consistent with the relic density $\Omega h^{2}=0.1198 \pm 0.0026$ within $3 \sigma$. We vary $C_{h, 3}$ from -0.7 to 0.7 and $\kappa$ from 0 to 0.75 to obtain Figure 2 . We also fix the coupling $C_{S, 3}=0.1$ in these plots.

In Figure 2(a), we vary the scalar DM mass between $55 \mathrm{GeV}$ and $65 \mathrm{GeV}$ and the fermion DM mass between 40 and $1000 \mathrm{GeV}$. In Figure 2(b), we take the variation of the fermionic DM mass between $55 \mathrm{GeV}$ and $65 \mathrm{GeV}$ and the scalar mass between 40 and $1000 \mathrm{GeV}$. We find that a large region in the parameter space satisfies the bound on WIMPnucleon cross section as imposed by the recent LUX-2016 and XENON-2017 experimental data. We find that the scalar DM mass $M_{S} \sim 60 \mathrm{Gev}$ provides the dominant contributions in the relic density. The contribution decreases with $M_{S}$. However, we need this scalar part to achieve the relic density as observed by the WMAP/Planck. In the second case, the fermionic contribution remains the same $(\sim 50 \%)$ in the region $55 \lesssim M_{S} \lesssim 65 \mathrm{GeV}$. We show these variations of the DM contribution in Figure 3 with the DM mass. The red points indicate the fermionic contribution whereas blue points stand for the scalar contribution to the correct relic density $\left(\Omega h^{2}=0.1198 \pm 0.0026\right)$ within $3 \sigma$.

\section{Conclusion}

In this paper, using two right-handed singlet fermions, we have explained the neutrino mass through type I seesaw mechanism. We have chosen three representative "benchmark points" of three different Majorana mass parameter spaces $\left(\sim 10^{3}, 10^{6}\right.$, and $\left.10^{12}\right)$ and particular structure of the Yukawa couplings matrix, that is, the Dirac mass matrix to explain the neutrino mass-squared differences as observed by the neutrino experiments. We have also calculated the PMNS mixing angles and the other low-energy variables, that is, nonunitary constraints on the PMNS matrix and LFV constraints from $\mu \rightarrow e \gamma$. The combinations of the new Yukawa couplings and the heavy neutrino mass satisfied the neutrino mass and mixing angles constraints [89].

In the presence of $Z_{2}$ and $Z_{2}^{\prime}$ symmetries, we have also analyzed the two-component Higgs portal self-annihilating dark matter particles. The regions of mass $65-550 \mathrm{GeV}$ of a Higgs portal fermionic or scalar dark matter models are excluded by the recent LUX experiment. In this model, we have shown that the regions of the parameter space with two-component dark matter particles are still allowed from direct search experiment and the WMAP/Planck data. For different fermionic Higgs portal coupling $C_{h, 3}$ and fermion dark matter mass, we have obtained viable scalar dark matter mass between $50 \mathrm{GeV}$ and $\sim 300 \mathrm{TeV}$. We have also obtained the similar region of fermionic dark matter mass for different scalar Higgs portal coupling $\kappa$. The unitary bounds are violated the dark matter mass above $300 \mathrm{TeV}$ [90]. Here, we do not intend to show that all the parameter spaces satisfy the experimental results. Rather, in the framework of our model, we have wanted to use the advantage of a two-component dark matter which has a large region of parameter spaces satisfying the constraint of various dark matter experiments.

The model ESSFSM is considered here to present the minimal seesaw mechanism and two-component dark matters in terms of particles content. This model can explain the observed tiny neutrino mass-squared differences and the mixing angles in oscillation experiments. The regions in the 
parameter space are also consistent with the relic density of dark matter observed by the Planck, WMAP experiments, and the recent null results of the WIMPs dark matter from the direct search LUX-2016 and XENON-2017 experiments.

\section{Conflicts of Interest}

The author declares that there are no conflicts of interest regarding the publication of this paper.

\section{Acknowledgments}

The authors would like to thank Subhendu Rakshit and Vishnudath K. N. for useful discussions.

\section{References}

[1] A. Aad, T. Abajyan, and B. Abbott, "Observation of a new particle in the search for the Standard Model Higgs boson with the ATLAS detector at the LHC," Physics Letters B, vol. 716, no. 1, pp. 1-29, 2012.

[2] P. P. Giardino, K. Kannike, I. Masina, M. Raidal, and A. Strumia, "The universal Higgs fit," Journal of High Energy Physics, vol. 2014, no. 5, 2014.

[3] C. Mariotti et al., "Observation of a new boson at a mass of 125 gev with the cms experiment at the lhc," Physics Letters B, vol. 716, no. 1, pp. 30-61, 2012.

[4] F. Rühr, "Prospects for BSM searches at the high-luminosity LHC with the ATLAS detector," Nuclear and Particle Physics Proceedings, vol. 273-275, pp. 625-630, 2016.

[5] P. A. R. Ade, N. Aghanim, M. Arnaud et al., "Planck 2015 results. XIII. Cosmological parameters," Astronomy \& Astrophysics, vol. 594, Article ID 201525830, 2016.

[6] E. Giusarma, M. Gerbino, O. Mena, S. Vagnozzi, S. Ho, and K. Freese, "Improvement of cosmological neutrino mass bounds," Physical Review D: Particles, Fields, Gravitation and Cosmology, vol. 94 , no. 8, 2016.

[7] S. Vagnozzi, E. Giusarma, O. Mena et al., "Unveiling $v$ secrets with cosmological data: neutrino masses and mass hierarchy," Physical Review D, vol. 96, no. 12, 2017.

[8] P. Minkowski, " $\mu \rightarrow \mathrm{e} \gamma$ at a rate of one out of $10^{9}$ muon decays?" Physics Letters B, vol. 67, pp. 421-428, 1977.

[9] M. Gell-Mann, P. Ramond, and R. Slansky, "Complex spinors and unified theories," in Proceedings of the Supergravity Workshop, pp. 315-318, 1979.

[10] T. R. Yanagida, in Proceedings of the Workshop on Unified Theory and Baryon Number in the Universe, pp. 95-98, 1979.

[11] R. N. Mohapatra and G. Senjanovic, "Neutrino mass and spontaneous parity nonconservation," Physical Review Letters, vol. 44 , p. $912,1980$.

[12] M. Mitra, G. Senjanović, and F. Vissani, "Neutrinoless double beta decay and heavy sterile neutrinos," Nuclear Physics B, vol. 856, no. 1, pp. 26-73, 2012.

[13] B. Karmakar and A. Sil, "Nonzero," Physical Review D: Particles, Fields, Gravitation and Cosmology, vol. 91, no. 1, 2015.

[14] N. Nath, M. Ghosh, S. Goswami, and S. Gupta, "Phenomenological study of extended seesaw model for light sterile neutrino," Journal of High Energy Physics, vol. 3, no. 75, pp. 1-29, 2017.

[15] S. M. Boucenna, S. Morisi, and J. W. F. Valle, "The low-scale approach to neutrino masses," Advances in High Energy Physics, vol. 2014, Article ID 831598, 15 pages, 2014.
[16] F. F. Deppisch, P. S. B. Dev, and A. Pilaftsis, "Neutrinos and collider physics," New Journal of Physics, vol. 17, no. 7, Article ID 075019, 2015.

[17] S. Khan, S. Goswami, and S. Roy, "Vacuum stability constraints on the minimal singlet TeV seesaw model," Physical Review D: Particles, Fields, Gravitation and Cosmology, vol. 89, no. 7, 2014.

[18] J. Chakrabortty, H. Z. Devi, S. Goswami, and S. Patra, "Neutrinoless double- $\beta$ decay in $\mathrm{TeV}$ scale left-right symmetric models," Journal of High Energy Physics, vol. 2012, no. 8, 2012.

[19] P. Ghosh, A. K. Saha, and A. Sil, "Study of electroweak vacuum stability from extended higgs portal of dark matter and neutrinos," High Energy Physics-Phenomenology, pp. 1-33, 2017.

[20] I. Garg, S. Goswami, K. Vishnudath, and N. Khan, "Electroweak vacuum stability in presence of singlet scalar dark matter in TeV scale seesaw models," Physical Review D: Particles, Fields, Gravitation and Cosmology, vol. 96, no. 5, 2017.

[21] S. Bhattacharya, S. Jana, and S. Nandi, "Neutrino masses and scalar singlet dark matter," Physical Review D: Particles, Fields, Gravitation and Cosmology, vol. 95, no. 5, 2017.

[22] N. Khan, Exploring Extensions of the Scalar Sector of the Standard Model [Ph.D. thesis], Indian Institute of Technology, Indore, India, 2017.

[23] E. Aprile et al., "Dark matter results from 225 live days of XENON100 Data," Physical Review Letters, vol. 109, no. 18, 2012.

[24] D. S. Akerib et al., "First results from the LUX dark matter experiment at the Sanford Underground Research Facility," Physical Review Letters, vol. 112, no. 9, 2014.

[25] D. S. Akerib, "Results from a search for dark matter in the complete LUX exposure," Physical Review Letters, vol. 118, no. 2, 2017.

[26] E. Aprile et al., "First dark matter search results from the xenonlt experiment," Physical Review Letters, vol. 119, no. 18, 2017.

[27] M. M. Ettefaghi and R. Moazzemi, "Analyzing of singlet fermionic dark matter via the updated direct detection data," The European Physical Journal C, vol. 77, no. 5, 2017.

[28] P. Athron, C. Balázs, T. Bringmann et al., "Status of the scalar singlet dark matter model," The European Physical Journal C, vol. 77, no. 568, 2017.

[29] N. Khan and S. Rakshit, "Study of electroweak vacuum metastability with a singlet scalar dark matter," Physical Review D: Particles, Fields, Gravitation and Cosmology, vol. 90, no. 11, 2014.

[30] A. Dutta Banik, M. Pandey, D. Majumdar, and A. Biswas, "Two component WIMP-FImP dark matter model with singlet fermion, scalar and pseudo scalar," The European Physical Journal C, vol. 77, no. 10, 2017.

[31] A. Albert, B. Anderson, K. Bechtol et al., "Searching for Dark Matter Annihilation in Recently Discovered Milky Way Satellites with Fermi-LAT,' The Astrophysical Journal, vol. 834, no. 2, 2017.

[32] D. Harvey, R. Massey, T. Kitching, A. Taylor, and E. Tittley, “The nongravitational interactions of dark matter in colliding galaxy clusters," Science, vol. 347, no. 6229, pp. 1462-1465, 2015.

[33] F. Kahlhoefer, K. Schmidt-Hoberg, J. Kummer, and S. Sarkar, "On the interpretation of dark matter self-interactions in Abell 3827," Monthly Notices of the Royal Astronomical Society, vol. 452, no. 1, pp. L54-L58, 2015.

[34] R. Campbell, S. Godfrey, H. E. Logan, A. D. Peterson, and A. Poulin, "Implications of the observation of dark matter selfinteractions for singlet scalar dark matter," Physical Review D: Particles, Fields, Gravitation and Cosmology, vol. 92, no. 5, 2015.

[35] S. Hannestad, A. Mirizzi, G. G. Raffelt, and Y. Y. Wong, "Neutrino and axion hot dark matter bounds after WMAP-7," Journal 
of Cosmology and Astroparticle Physics, vol. 2010, no. 08, pp. 001001, 2010.

[36] K. J. Bae, H. Baer, and E. J. Chun, "Mixed axion/neutralino dark matter in the SUSY DFSZ axion model," Journal of Cosmology and Astroparticle Physics, vol. 2013, no. 12, pp. 028-028, 2013.

[37] E. Ma, "Verifiable radiative seesaw mechanism of neutrino mass and dark matter," Physical Review D: Particles, Fields, Gravitation and Cosmology, vol. 73, Article ID 077301, 2006.

[38] K. M. Zurek, "Multi-Component Dark Matter," Physical Review $D$, vol. 79, no. 11, Article ID 115002, 2009.

[39] B. Batell, "Dark discrete gauge symmetries," Physical Review D: Particles, Fields, Gravitation and Cosmology, vol. 83, no. 3, 2011.

[40] H. Fukuoka, D. Suematsu, and T. Toma, "Signals of dark matter in a supersymmetric two dark matter model," Journal of Cosmology and Astroparticle Physics, vol. 2011, no. 07, pp. 001-001, 2011.

[41] G. Belanger, K. Kannike, A. Pukhov, and M. Raidal, "Impact of semi-annihilations on dark matter phenomenology. An example of $\mathrm{Z}_{N}$ symmetric scalar dark matter," Journal of Cosmology and Astroparticle Physics, 2012.

[42] M. Aoki, M. Duerr, J. Kubo, and H. Takano, "Multicomponent dark matter systems and their observation prospects," Physical Review D: Particles, Fields, Gravitation and Cosmology, vol. 86, no. 7, 2012.

[43] I. P. Ivanov and V. Keus, Physical Review D: Particles, Fields, Gravitation and Cosmology, vol. 86, no. 1, 2012.

[44] D. Chialva, P. S. Dev, and A. Mazumdar, "Multiple dark matter scenarios from ubiquitous stringy throats," Physical Review D: Particles, Fields, Gravitation and Cosmology, vol. 87, no. 6, 2013.

[45] J. Heeck and H. Zhang, "Exotic charges, multicomponent dark matter and light sterile neutrinos," Journal of High Energy Physics, vol. 5, no. 164, 2013.

[46] K. P. Modak, D. Majumdar, and S. Rakshit, "A possible explanation of low energy $\gamma$-ray excess from galactic centre and Fermi bubble by a Dark Matter model with two real scalars," Journal of Cosmology and Astroparticle Physics, vol. 2015, no. 03, pp. 011011, 2015.

[47] M. Aoki, J. Kubo, and H. Takano, “ Two-loop radiative seesaw mechanism with multicomponent dark matter explaining the possible ", Physical Review D: Particles, Fields, Gravitation and Cosmology, vol. 87, no. 11, 2013.

[48] C. Geng, D. Huang, and L. Tsai, "Imprint of multicomponent dark matter on AMS-02," Physical Review D: Particles, Fields, Gravitation and Cosmology, vol. 89, no. 5, 2014.

[49] Y. Kajiyama, H. Okada, and T. Toma, "Multicomponent dark matter particles in a two-loop neutrino model," Physical Review D: Particles, Fields, Gravitation and Cosmology, vol. 88, no. 1, 2013.

[50] S. Bhattacharya, A. Drozd, B. Grzadkowski, and J. Wudka, "Two-component dark matter," Journal of High Energy Physics, vol. 2013, no. 10, 2013.

[51] A. Biswas, D. Majumdar, A. Sil, and P. Bhattacharjee, "Two component dark matter: a possible explanation of $130 \mathrm{GeV} \gamma$ ray line from the galactic centre," Journal of Cosmology and Astroparticle Physics, 2013.

[52] A. Biswas, D. Majumdar, and P. Roy, "Nonthermal two component dark matter model for Fermi-LAT $\gamma$-ray excess and 3.55 keV X-ray line," Journal of High Energy Physics, vol. 2015, no. 65, 2015.

[53] L. Bian, T. Li, J. Shu, and X. Wang, “Two component dark matter with multi-Higgs portals," Journal of High Energy Physics, vol. 2015, no. 3, 2015.
[54] S. Bhattacharya, A. Drozd, B. Grzadkowski, and J. Wudka, "Constraints on Two-component Dark Matter," Acta Physica Polonica B, vol. 44, no. 11, p. 2373, 2013.

[55] L. Bian, R. Ding, and B. Zhu, "Two component Higgs-portal dark matter," Physics Letters B, vol. 728, pp. 105-113, 2014.

[56] P. Gu, "Multi-component dark matter with magnetic moments for Fermi-LAT gamma-ray line," Physics of the Dark Universe, vol. 2, no. 1, pp. 35-40, 2013.

[57] A. DiFranzo and G. Mohlabeng, "Multi-component dark matter through a radiative Higgs portal," Journal of High Energy Physics, vol. 2017, no. 1, 2017.

[58] S. Bhattacharya, P. Ghosh, and P. Poulose, "Multipartite interacting scalar dark matter in the light of updated LUX data," Journal of Cosmology and Astroparticle Physics, vol. 2017, no. 04, pp. 043-043, 2017.

[59] A. Chaudhuri, N. Khan, B. Mukhopadhyaya, and S. Rakshit, "Dark matter candidate in an extended type III seesaw scenario," Physical Review D: Particles, Fields, Gravitation and Cosmology, vol. 91, no. 5, 2015.

[60] J. A. Casas and A. Ibarra, "Oscillating neutrinos and muon $\rightarrow$ e, gamma," Nuclear Physics B, vol. 618, no. 1-2, Article ID 0103065, pp. 171-204, 2001.

[61] G. Bambhaniya, P. Bhupal Dev, S. Goswami, S. Khan, and W. Rodejohann, "Naturalness, vacuum stability, and leptogenesis in the minimal seesaw model," Physical Review D: Particles, Fields, Gravitation and Cosmology, vol. 95, no. 9, 2017.

[62] F. Capozzi, E. Lisi, A. Marrone, D. Montanino, and A. Palazzo, "Neutrino masses and mixings: Status of known and unknown 3 v parameters," Nuclear Physics B, vol. 908, pp. 218-234, 2016.

[63] I. Esteban, M. C. Gonzalez-Garcia, M. Maltoni, I. MartinezSoler, and T. Schwetz, "Updated fit to three neutrino mixing: exploring the accelerator-reactor complementarity," Journal of High Energy Physics, vol. 2017, no. 1, 2017.

[64] Z. Z. Xing and S. Zhou, "Why is the $3 \times 3$ neutrino mixing matrix almost unitary in realistic seesaw models?" High Energy Physics and Nuclear Physics, vol. 30, pp. 828-830, 2006.

[65] W. Grimus and L. Lavoura, "The seesaw mechanism at arbitrary order: disentangling the small scale from the large scale," Journal of High Energy Physics, vol. 11, no. 42, 2000.

[66] G. Bélanger, F. Boudjema, A. Pukhov, and A. Semenov, "MicrOMEGAs4.1: Two dark matter candidates," Computer Physics Communications, vol. 192, pp. 322-329, 2015.

[67] B. W. Lee, C. Quigg, and H. B. Thacker, "Weak interactions at very high energies: the role of the Higgs-boson mass," Physical Review D: Particles, Fields, Gravitation and Cosmology, vol. 16, no. 5, pp. 1519-1531, 1977.

[68] G. Cynolter, E. Lendvai, and G. Pocsik, "Note on unitarity constraints in a model for a singlet scalar dark matter candidate," Acta Physica Polonica B, vol. 36, Article ID 0410102, pp. 827832, 2005.

[69] Y. P. Yao and C. P. Yuan, "Modification of the equivalence theorem due to loop corrections," Physical Review D: Particles, Fields, Gravitation and Cosmology, vol. 38, no. 7, pp. 2237-2244, 1988.

[70] H. G. J. Veltman, “The equivalence theorem," Physical Review D: Particles, Fields, Gravitation and Cosmology, vol. 41, no. 7, pp. 2294-2311, 1990.

[71] H. He, Y. Kuang, and X. Li, "On the precise formulation of the equivalence theorem," Physical Review Letters, vol. 69, no. 18, pp. 2619-2622, 1992. 
[72] S. Antusch and O. Fischer, "Non-unitarity of the leptonic mixing matrix: present bounds and future sensitivities," Journal of High Energy Physics, vol. 2014, no. 10, article 94, 2014.

[73] S. Antusch and O. Fischer, "Probing the nonunitarity of the leptonic mixing matrix at the CEPC," International Journal of Modern Physics A, vol. 31, no. 33, p. 1644006, 2016.

[74] P. Achard et al., "Search for heavy isosinglet neutrino in $\mathrm{e}^{+} \mathrm{e}^{-}$annihilation at LEP," Physics Letters $B$, vol. 517, no. 1-2, Article ID 0107014, pp. 67-74, 2001.

[75] O. Adriani et al., "Search for isosinglet neutral heavy leptons in Z0 decays," Phys. Lett, vol. 95, pp. 371-382, 1992.

[76] P. Abreu, W. Adam, and T. Adye, "Search for neutral heavy leptons produced in Z decays," Zeitschrift für Physik C, vol. 74, no. 1, pp. 57-71, 1997.

[77] J. Grifols and A. Méndez, "Electromagnetic properties of the tau lepton from Z0 decay," Physics Letters B, vol. 255, no. 4, pp. 611612, 1991.

[78] A. M. Baldini, Y. Bao, E. Baracchini et al., "Search for the lepton flavour violating decay $\mu^{+} \rightarrow \mathrm{e}^{+} \gamma$ with the full dataset of the MEG experiment," The European Physical Journal C, 2016.

[79] D. Tommasini, G. Barenboim, J. Bernabéu, and C. Jarlskog, "Non-decoupling of heavy neutrinos and lepton flavour violation," Nuclear Physics B, vol. 444, no. 3, pp. 451-467, 1995.

[80] G. Bélanger, B. Dumont, U. Ellwanger, J. F. Gunion, and S. Kraml, "Global fit to Higgs signal strengths and couplings and implications for extended Higgs sectors," Physical Review D: Particles, Fields, Gravitation and Cosmology, vol. 88, no. 7, 2013.

[81] G. Aad, B. Abbott, J. Abdallah et al., "Measurements of the Higgs boson production and decay rates and constraints on its couplings from a combined ATLAS and CMS analysis of the LHC pp collision data at $\mathrm{s}=7$ and $8 \mathrm{TeV}$," Journal of High Energy Physics, vol. 8, no. 54, 2016.

[82] P. A. R. Ade, N. Aghanim, and C. Armitage-Caplan, "Planck 2013 results. XVI. Cosmological parameters," Astronomy \& Astrophysics, vol. 571, Article ID 201321591, 2014.

[83] B. Batell, M. Pospelov, and A. Ritz, "Direct detection of multicomponent secluded WIMPs," Physical Review D: Particles, Fields, Gravitation and Cosmology, vol. 79, no. 11, 2009.

[84] S. Profumo, K. Sigurdson, and L. Ubaldi, "Can we discover dualcomponent thermal WIMP dark matter?" Journal of Cosmology and Astroparticle Physics, vol. 2009, no. 12, pp. 016-016, 2009.

[85] J. Herrero-Garcia, A. Scaffidi, M. White, and A. G. Williams, "On the direct detection of multi-component dark matter: sensitivity studies and parameter estimation," Journal of Cosmology and Astroparticle Physics, vol. 2017, no. 11, pp. 021-021, 2017.

[86] A. Alloul, N. D. Christensen, C. Degrande, C. Duhr, and B. Fuks, "FeynRules 2.0-A complete toolbox for tree-level phenomenology," Computer Physics Communications, vol. 185, no. 8, pp. 2250-2300, 2014.

[87] L. Covi, F. Roulet, and F. Vissani, "CP violating decays in leptogenesis scenarios," Physics Letters B, vol. 384, no. 1-4, pp. 169174, 1996.

[88] J. A. Harvey and M. S. Turner, "Cosmological baryon and lepton number in the presence of electroweak fermion-number violation," Physical Review D: Particles, Fields, Gravitation and Cosmology, vol. 42, p. 3344, 1990.

[89] A. Halprin, P. Minkowski, H. Primakoff, and S. P. Rosen, "Double-beta decay and a massive Majorana neutrino," Physical Review D: Particles, Fields, Gravitation and Cosmology, vol. 13, no. 9, pp. 2567-2571, 1976.
[90] K. Griest and M. Kamionkowski, "Unitarity limits on the mass and radius of dark-matter particles," Physical Review Letters, vol. 64, no. 6, pp. 615-618, 1990. 

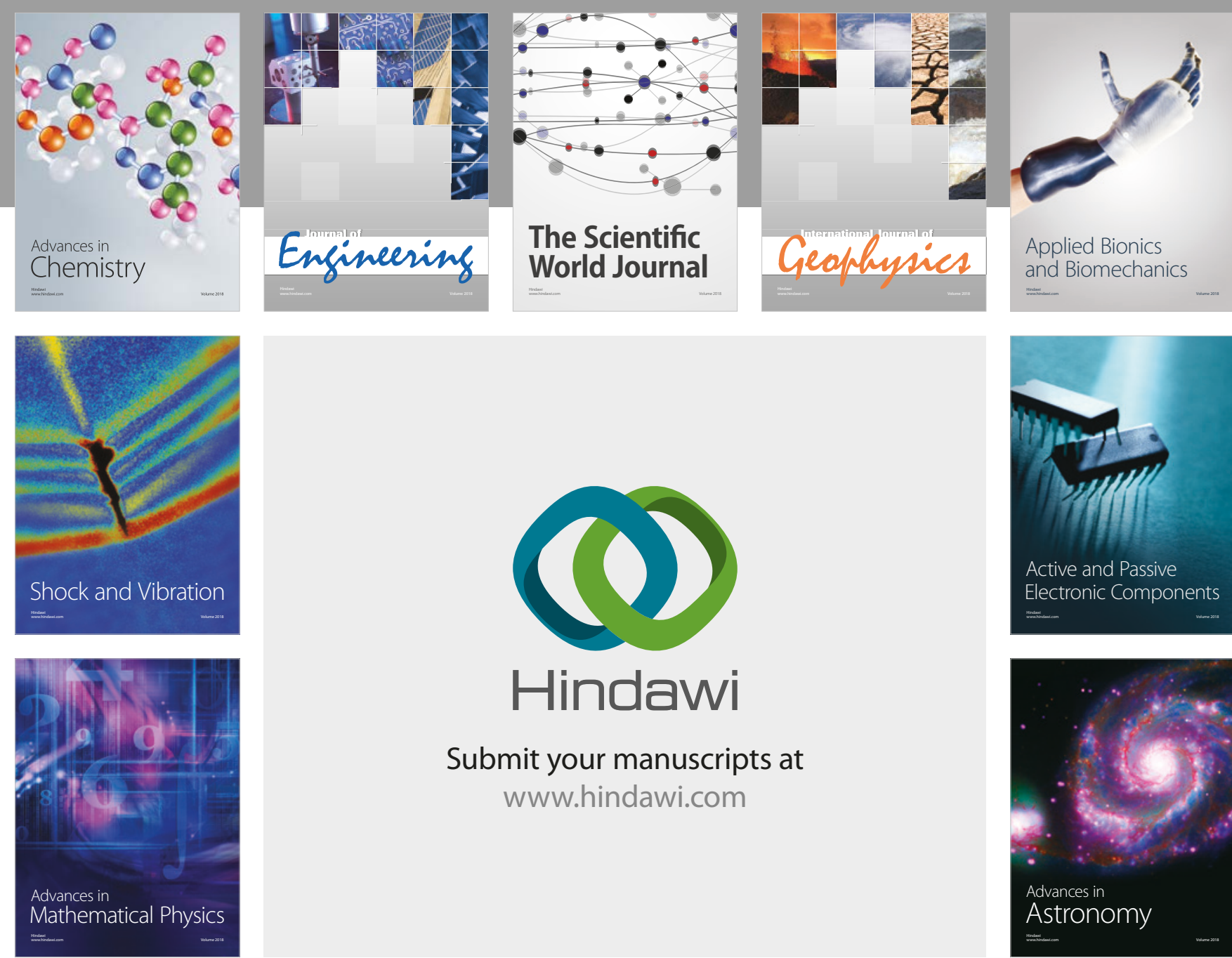

Submit your manuscripts at

www.hindawi.com

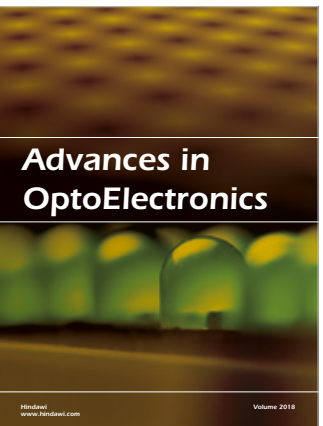

\section{Rotcting Machinery}
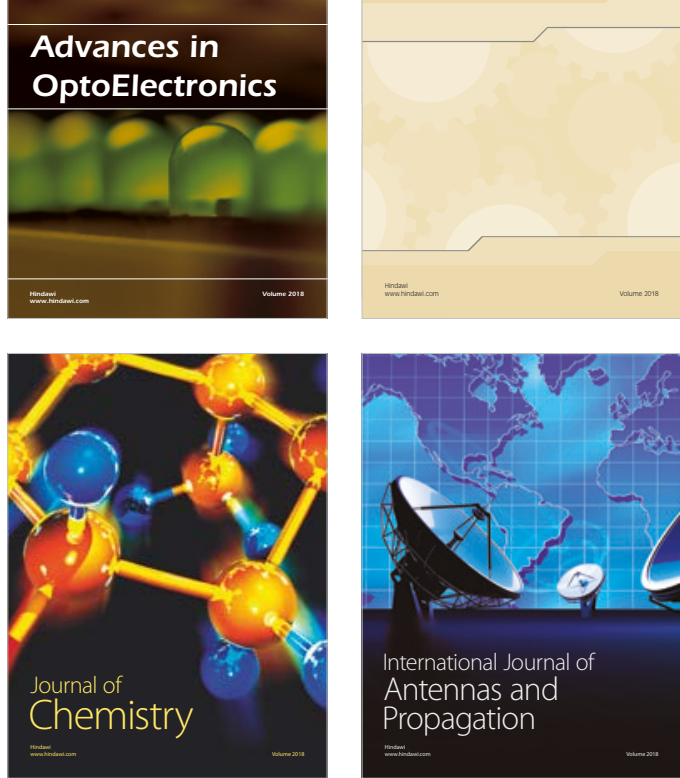

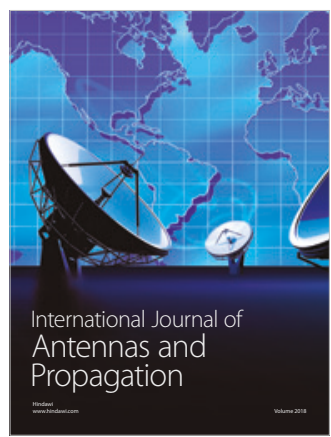

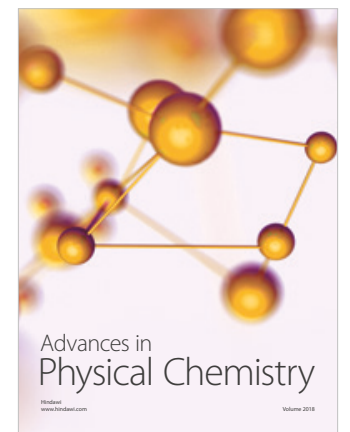

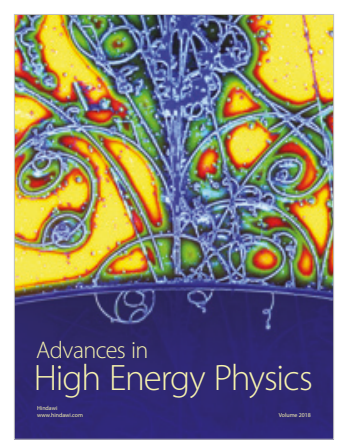

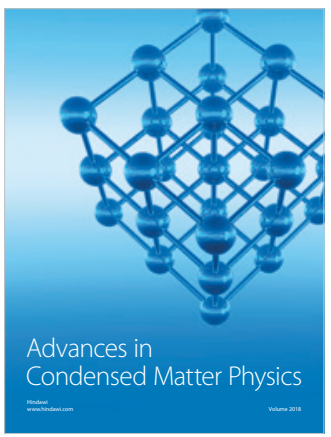

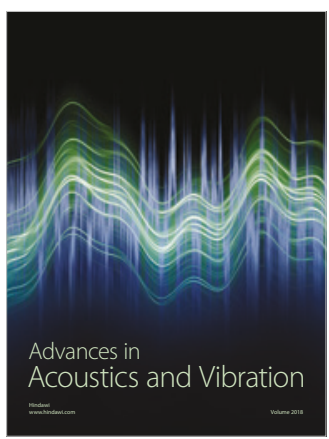

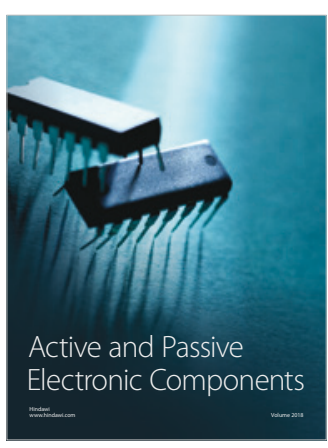
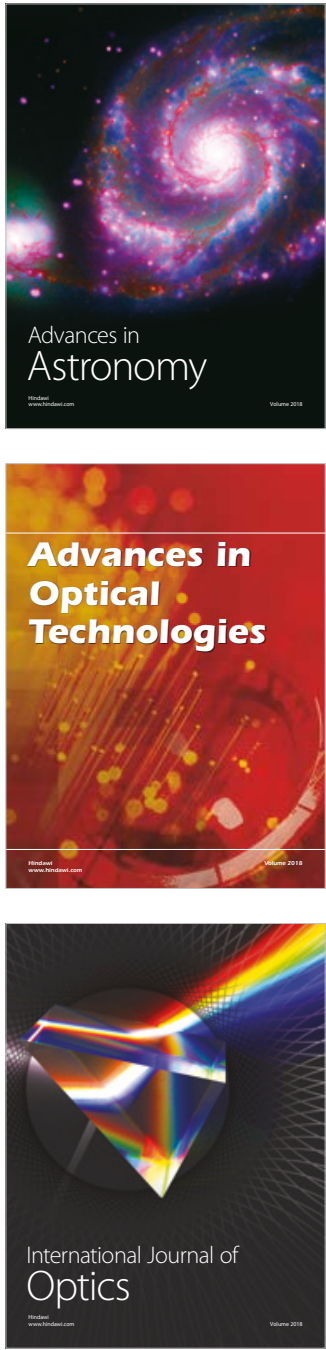